\title{
VOCAL LEARNING IN BATS: FROM GENES TO BEHAVIOUR
}

\author{
SONJA C. VERNES ${ }^{* 1,2}$ \\ *Corresponding Author: sonja.vernes@mpi.nl \\ ${ }^{1}$ Neurogenetics of Vocal Communication Group, Max Planck Institute for \\ Psycholinguistics, Nijmegen, The Netherlands \\ ${ }^{2}$ Donders Institute for Brain, Cognition and Behaviour, Nijmegen, The Netherlands
}

\section{Vocal learning}

Vocal production learning (herein 'vocal learning') - the ability to modify vocal signals based on conspecific auditory feedback - is an essential component of human spoken language (Bolhuis, Okanoya, \& Scharff, 2010a; Nowicki \& Searcy, 2014). Infants learning to speak must perform this task, which includes auditory perception, memorization of template, vocal motor planning and production, template matching to determine goodness of fit and modification of the vocal-motor output as necessary (Petkov \& Jarvis, 2012). The complexity of this task suggests that multiple neurobiological and genetic mechanisms are likely to underlie its evolution and biological encoding. Given the necessary limitations of studying vocal learning in humans, animal models represent an opportunity to understand the neurogenetic mechanisms underlying this spoken languagerelevant trait.

\subsection{Animal models of vocal learning}

Vocal learning is a rare trait in the animal kingdom. Few non-human species have convincingly shown this trait. To date, vocal learning has been documented in some cetacean, pinniped, elephant, bat and bird species (Bolhuis, Okanoya, \& Scharff, 2010b; Janik \& Slater, 1997). Songbirds have dominated the study of vocal learning due to their well-defined learning paradigm, sexual dimorphism of their song, their ease of handling and ability to breed in captivity (Bolhuis et al., 2010b; Condro \& White, 2014). Mammalian vocal learning has, by comparison, been understudied. Sea mammal vocal learning has been most well documented 
from this group (Janik \& Slater, 2000; Janik \& Slater, 1997), however due to their size and habitat they present hurdles for neurological or genetic interrogations.

\section{Bat models of vocal learning}

Bats are highly social animals that have developed sophisticated vocal systems for navigation and communication (Vernes, 2017). Social communication in bats is often facilitated by low frequency calls (in the hearing range) and in some species these calls show evidence that they may be learned (Knörnschild, 2014). Two species with evidence for learned calls, Phyllostomus discolour and Rousettus aegyptiacus, can be maintained in laboratory colonies, making them amenable to neurogenetic manipulations (Esser, 1994; Knörnschild, 2014; Prat, Taub, \& Yovel, 2015). Thus, their small size, ability to breed in captivity and sophisticated vocal communications, make bats an exciting mammalian model for the study of vocal learning.

\subsection{Studying vocal learning behaviour in bats}

It is essential to have a well-defined and highly controlled behavioural paradigm to dissect out the neuro-genetic mechanisms underlying vocal learning. We are developing a vocal learning paradigm in which bats are induced to learn novel vocalisations via an automated playback design. To date volitional control of vocal production and vocal plasticity (usage learning) has been demonstrated.

\subsection{Studying the neurogenetics of vocal learning in bats}

Multiple approaches are being used to understand the neurogenetics contributing to vocal behaviour. Activation of neurons in response to perception or production of communication calls is being mapped to determine the brain regions involved. To facilitate evolutionary comparisons and molecular genetic studies, de novo sequencing of $P$. discolour and $R$. aegyptiacus genomes are being performed. To understand the role of language-related genes, the expression patterns of FoxP2, FoxP1 and CntnaP2 have been comprehensively mapped in the brains of both species. Finally, gene knockdown studies will determine the contribution of genes such as FoxP2 to vocal behavior.

Bringing together genetic, neurobiological and behavioural studies in this way will shed light on the encoding of vocal learning in bats, and ultimately inform our understanding of the evolution of this language-relevant trait. 


\section{Acknowledgements}

This work was funded by a Marie Curie Career Integration Grant (PCIG12-GA2012-333978), and a Max Planck Research Group Grant both awarded to S.C.V. This work was also funded by a Human Frontiers Science Program (HFSP) Research grant (RGP0058/2016) and is part of a collaborative project involving Prof. Lutz Wiegrebe (LMU, Germany), Prof Michael Yartsev (UC Berkeley, USA) and Prof Uwe Firzlaff (TUM, Germany). With thanks to Ella Lattenkamp, Janine Mengede, Ine Alvarez van Tussenbroek, Laura Baas and Ksenia Lavrichenko for their contributions to this project.

\section{References}

Bolhuis, J. J., Okanoya, K., \& Scharff, C. (2010a). Twitter evolution: converging mechanisms in birdsong and human speech. Nat Rev Neurosci, 11(11), 747-759. doi:10.1038/nrn2931

Condro, M. C., \& White, S. A. (2014). Recent Advances in the Genetics of Vocal Learning. Comparative cognition \& behavior reviews, 9, 75-98. doi:10.3819/ccbr.2014.90003

Esser, K. H. (1994). Audio-vocal learning in a non-human mammal: the lesser spear-nosed bat Phyllostomus discolor. Neuroreport, 5(14), 1718-1720.

Janik, V. M., \& Slater, P. J. (2000). The different roles of social learning in vocal communication. Animal behaviour, 60(1), 1-11. doi:10.1006/anbe. 2000.1410

Janik, V. M., \& Slater, P. J. B. (1997). Vocal learning in mammals. Advances in the Study of Behavior, Vol 26, 26, 59-99. doi:Doi 10.1016/S00653454(08)60377-0

Knörnschild, M. (2014). Vocal production learning in bats. Current opinion in neurobiology, 28, 80-85. doi:10.1016/j.conb.2014.06.014

Nowicki, S., \& Searcy, W. A. (2014). The evolution of vocal learning. Current opinion in neurobiology, 28, 48-53. doi:10.1016/j.conb.2014.06.007

Petkov, C. I., \& Jarvis, E. D. (2012). Birds, primates, and spoken language origins: behavioral phenotypes and neurobiological substrates. Frontiers in evolutionary neuroscience, 4, 12. doi:10.3389/fnevo.2012.00012

Prat, Y., Taub, M., \& Yovel, Y. (2015). Vocal learning in a social mammal: Demonstrated by isolation and playback experiments in bats. Science Advances, 1(2), e1500019. doi:10.1126/sciadv.1500019

Vernes, S. C. (2017). What bats have to say about speech and language. Psychonomic Bulletin \& Review, 24(1), 111-117. doi:10.3758/s13423016-1060-3 